\title{
Suppressing the rejection of pancreatic tumours
}

The immune system is often co-opted by developing tumours to promote an immunosuppressive microenvironment that prevents tumour rejection. This is particularly evident for pancreatic ductal adenocarcinoma (PDA), which initially develops as pancreatic intraepithelial neoplasia (PanIN; a premalignant lesion) and is associated with the infiltration of immunosuppressive leukocytes. Two papers in Cancer Cell now characterize a mechanism of immunosuppression in pancreatic tumorigenesis.

To investigate immunomodulation during the development of PDA, Bayne and colleagues used KPC genetically engineered mice, which have pancreas-specific Kras ${ }^{\mathrm{G} 12 \mathrm{D}}$ and $\operatorname{Trp} 53^{\mathrm{R} 172 \mathrm{H}}$ mutations. Pylayeva-Gupta and colleagues used an allograft model in which green fluorescent protein (GFP) ${ }^{+}$pancreatic ductal epithelial cells (PDECs) expressing mutant KRAS (KRAS-G12D) were injected into the pancreata of syngeneic mice. Both mouse models developed PanINs and PDAs with features of the human disease. In particular, the tumours in both models were infiltrated by myeloid-derived suppressor cells (MDSCs) $\mathrm{GR} 1^{+} \mathrm{CD} 11 \mathrm{~b}^{+}$cells that are thought to suppress $T$ cell-mediated antitumour immune responses. Bayne and colleagues found that MDSCs from KPC mice suppressed T cell proliferation and interferon- $\gamma$ production in vitro. These authors noted that KPC mice developed splenomegaly as MDSCs and their $\mathrm{KIT}^{+}$

MDSCs are recruited by KRAS-G12Dtransformed pancreatic tumour cells ... to suppress $\mathrm{CD} 8{ }^{+} \mathrm{T}$ cellmediated antitumour immune responses precursors accumulated in the spleen, indicating that a tumour-derived factor might promote the development of MDSCs. Indeed, they found that culturing $\mathrm{KIT}^{+}$ splenocytes isolated from tumour-bearing KPC mice with conditioned media from PDA cells promoted their proliferation and the expression of GR1 and CD11b; these cells suppressed $\mathrm{T}$ cell proliferation in vitro.

Both groups looked for a tumour cell-derived secreted factor that could be responsible for the splenomegaly and the recruitment of MDSCs to PanINs and PDAs. Analyses of supernatants from allograft tumour tissue and from the culture of a panel of pancreatic tumour cell lines identified consistently high expression of granulocytemacrophage colony-stimulating factor (GM-CSF). Human PanIN and PDA tumour cells also expressed GM-CSF. Pylayeva-Gupta and colleagues found that other types of pancreatic lesion that are not associated with oncogenic
KRAS did not express GM-CSF. Transcripts of Csf2 (which encodes GM-CSF) were also increased in GFP+ KRAS-G12D PDECs, which indicates that KRAS-G12D causes the upregulation of GM-CSF expression. This cytokine induced the proliferation and differentiation into MDSCs of bone marrow-derived haematopoietic precursor cells isolated from the allograft mouse model and of $\mathrm{KIT}^{+}$splenocytes from KPC mice.

Does GM-CSF secretion by pancreatic tumour cells promote an immunosuppressive microenvironment in vivo? Bayne and colleagues implanted PDA1 cells (a PDA cell line that secretes GM-CSF) together with a neutralizing GM-CSF-specific antibody into wild-type mice and found that the cells failed to grow and that the implant sites appeared necrotic. Moreover, the number of infiltrating $\mathrm{CD} 45^{+} \mathrm{GR} 1^{+} \mathrm{CD} 11 \mathrm{~b}^{+}$cells at these sites was substantially decreased compared with sites of control PDA1 cell implantation; depletion of $\mathrm{GR} 1{ }^{+} \mathrm{CD} 11 \mathrm{~b}^{+}$cells also inhibited the growth of PDA1 cells in vivo. Pylayeva-Gupta and colleagues knocked down GM-CSF expression in GFP+ KRAS-G12D PDECs, which reduced their engraftment and specifically decreased the infiltration of GR $1^{+} \mathrm{CD} 11 b^{+}$cells. These GFP ${ }^{+}$PDECs were absent from the graft sites 2 weeks after implantation, corresponding with the infiltration of $\mathrm{CD} 8^{+} \mathrm{T}$ cells, which indicates that GM-CSF-mediated recruitment of MDSCs probably occurs when $T$ cells begin to infiltrate graft sites. The two groups showed that implanting either PDA1 cells with the GM-CSF-specific antibody or GFP+ KRAS-G12D PDECs with knocked down GM-CSF expression into mice lacking $\mathrm{CD}^{+} \mathrm{T}$ cells restored engraftment but did not rescue the infiltration of $G R 1^{+} C D 11 b^{+}$cells.

Together, these papers show that GR $1^{+} \mathrm{CD} 11 \mathrm{~b}^{+}$MDSCs are recruited by KRAS-G12D-transformed pancreatic tumour cells in the early stages of tumour development to suppress $\mathrm{CD}^{+} \mathrm{T}$ cell-mediated antitumour immune responses and thereby allow tumour growth.

Gemma K. Alderton, Senior Editor, Nature Reviews Cancer

This article is modified from the original in Nature Rev. Cancer (doi:10.1038/nrc3329)

ORIGINAL RESEARCH PAPERS Bayne, L. J. et al. Tumor-derived granulocytemacrophage colony-stimulating factor regulates myeloid inflammation and T cell immunity in pancreatic cancer. Cancer Cell 21, 822-835 (2012)| PylayevaGupta, Y. et al. Oncogenic Kras-induced GM-CSF production promotes the development of pancreatic neoplasia. Cancer Cell 21, 836-847 (2012) 